\title{
Usefulness of a Second Temporary Vena Cava Filter for Preventing Acute Pulmonary Thromboembolism
}

\author{
Tetsuya Ishikawa, MD; Teruo Okabe, MD; Kazuo Ogawa, MD; Jun Fuse, MD; \\ Yoko Oshiba, MD*; Akira Ono, MD**; Masao Chino, MD
}

\begin{abstract}
The usefulness of a temporary inferior vena cava filter (t-IVC-f) for the prevention of acute pulmonary thromboembolism (APTE) associated with venous thromboembolism (VTE) has been established, but not the requirement for an additional therapeutic method when the t-IVC-f has captured a thrombus. A woman underwent implantation of a t-IVC-f just caudal to the bifurcation of the renal vein immediately before cesarean section (C/S) for VTE that had occurred during the third trimester of pregnancy. After receiving a combination of anticoagulant and fibrinolytic therapies after the C/S, the t-IVC-f was found to contain a thrombus and in order to prevent its dissemination during the removal of the closed filter, another filter was inserted and expanded proximal to the first. Insertion of an additional t-IVC-f may prevent occurrence of APTE during removal of the primary filter containing a friable thrombus after fibrinolytic therapy, and the technique proved useful in the present case of VTE during pregnancy. (Circ J 2003; 67: 718-720)
\end{abstract}

Key Words: Acute pulmonary thromboembolism; Inferior vena cava; Oral anticoagulants; Pregnancy; Temporary filter; Venous thromboembolism

$\mathbf{T}$ he incidence of acute pulmonary thromboembolism (APTE) has been increasing in Japan, although it is still rare compared with Western countries? To prevent APTE becoming life threatening, the insertion of a temporary inferior vena cava filter (t-IVC-f) has been advocated for cases of venous thromboembolism (VTE) of the lower extremities? However, when the t-IVC-f captures a thrombus, other problems need to be anticipated, such as dispersal of thrombi after combination anticoagulant and fibrinolytic therapy, after thromboembolectomy or when removing the filter.

We present a case of VTE that occurred during the third trimester of pregnancy for which we used a primary and secondary t-IVC-f.

\section{Case Report}

A 29-year-old woman reported worsening of a sub-acute pain in the left anterior region of the thigh and edema (on the 1st day of the 31st week of pregnancy), and she was hospitalized with the suspicion of VTE of the left lower extremity. She did not have anamnesis.

Ultrasound examination showed dilatation of the left femoral vein in comparison with the right, and VTE was diagnosed by compression ultrasonography4 Anticoagulant therapy $(17,000$ units of standard heparin, once daily) was immediately begun with intermittent positive compression of the lower extremity. The serum protein-S concentration was at the lowest standard limit of $46 \%$. Pelvic computed tomography (CT) detected a thrombotic occlusion extend-

(Received October 24, 2001; revised manuscript received February 12, 2002; accepted March 1, 2002)

Departments of Cardiology, *Obstetrics and Gynecology, and **Anesthesiology, National Tokyo Medical Center, Tokyo, Japan

Mailing address: Tetsuya Ishikawa, MD, Department of Cardiology, The Jikei University School of Medicine, 3-19-18 Nishishinbashi, Minato-ku, Tokyo 105-8461, Japan. E-mail: ishikawa@jikei.ac.jp ing from the left common iliac vein to the femoral vein (Fig 1A,B). Because it was essential to prevent APTE, she had a cesarean section $(\mathrm{C} / \mathrm{S})$ on the 3rd day of the $32 \mathrm{nd}$ week of pregnancy. The standard heparin was changed to low-molecular-weight heparin (dalteparin) before the C/S with concommitant oral administration of low-dose aspirin. A t-IVC-f (Neuhaus Protect, Toray Medical Co Ltd, Tokyo, Japan) was inserted from the right subclavian vein and placed beneath the bifurcation of the renal vein immediately before the $\mathrm{C} / \mathrm{S}$ and expanded immediately after (approx $2 \mathrm{~h}$ later) because the immediate postpartum period after $\mathrm{C} / \mathrm{S}$ also carries a high risk for APTE4

On Day 8 of t-IVC-f retention, complete thrombotic occlusion and development of collateral circulation were detected by venography of the lower extremity (Fig 2). Pul-

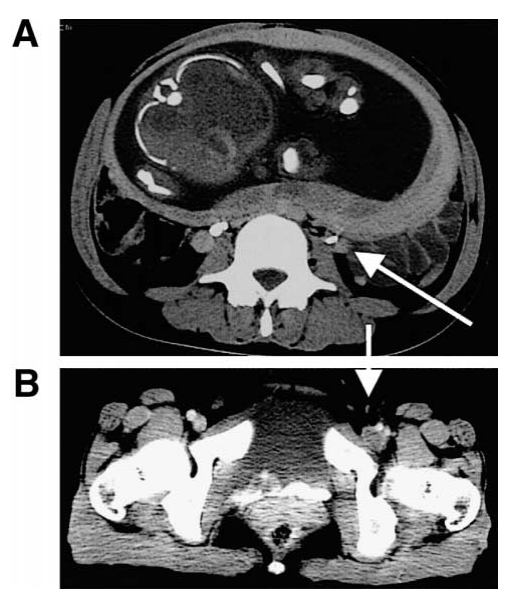

Fig 1. Pelvic computed tomography (CT) detected the venous thrombotic occlusion. (A) Left common iliac vein (arrow) was compressed by the uterus. Note the fetus. (B) Thrombotic occlusion of the left femoral vein (arrow). 


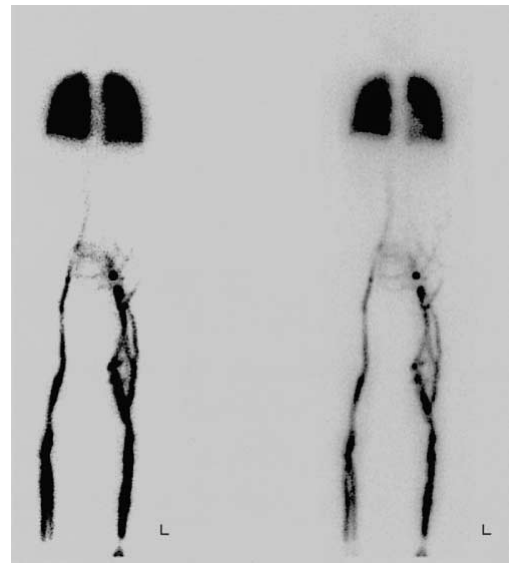

Fig 2. Venography of the lower extremity and pulmonary lung perfusion scintigraphy on Day 8 after t-IVC-f insertion. Complete thrombotic occlusion of the left femoral vein and development of collateral circulation can be seen. Pulmonary lung perfusion scintigraphy did not show any defects.

monary lung perfusion scintigraphy did not show any defects (Fig 2). On Day 10, a captured thrombus was seen by venography of the IVC (Fig 3A) and so fibrinolytic therapy with urokinase $(240,000$ units, once daily) and warfarin therapy were initiated. On Day 15 , although venography did not show a friable thrombus and despite contraction of the captured thrombus (Fig 3B), the VTE of her leg remained unchanged. therefore, we decided to remove the t-IVC-f with its captured thrombus and at the same time insert a second t-IVC-f from the right internal carotid vein, which was also expanded at the bifurcation of the renal vein (Fig 3C). The primary t-IVC-f was closed and then both of the filters were slowly removed. There were not any clinically suspicious symptoms related to APTE nor was a right-heart strain pattern detected on either standard 12-lead ECG or on cardiac ultrasound during her hospitalization.

Histopathological examination revealed that all the redcolored thrombi in the primary t-IVC-f, which remained in situ for 15 days, were several centimeters in size, accompanied by endothelial cells, and partial organization was seen, which was suspected to have occurred within a few days after formation of thrombi. The thrombus in the second tIVC-f showed similar histological features and was friable, so it could have lead to symptomatic APTE.

Clinically suspicious symptoms related to APTE have not been observed during the 15-month follow-up, but because her left leg is still edematous, oral anticoagulant therapy with warfarin has been maintained.

\section{Discussion}

In the present case involving a pregnant woman who had VTE in the third trimester, APTE was prevented at $\mathrm{C} / \mathrm{S}$ by using a t-IVC-f and then inserting a second filter at the time of removing the primary device in order to capture any friable thrombi dispersed during the removal of the closed t-IVC-f. In addition, APTE was further prevented by approximately 15 months of continuous oral anticoagulant therapy.

The patient had been suffering from pain in the left lower extremity and had a high possibility of VTE on ultrasound examination, 4 which was confirmed by pelvic CT

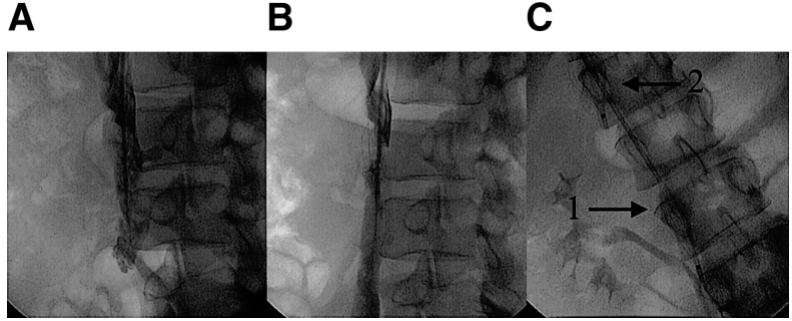

Fig 3. Venography of the IVC after t-IVC-f implantation. (A) On Day 10 after t-IVC-f insertion there is a captured thrombus in the filter. (B) On Day 15 after insertion and after fibrinolytic therapy with urokinase and warfarin there is almost no change in the captured thrombus. (C) Insertion of the second t-IVC-f immediately before removing the existing t-IVC-f with the captured thrombus $(\rightarrow$ indicates the existing filter as 1 , second t-IVC-f $\rightarrow$ indicated as 2) and expanded at the bifurcation of the renal vein. The primary t-IVC-f was closed and both filters were gradually pulled and removed.

that showed a thrombus extending from the left common iliac vein to the femoral vein (Fig 1A,B). Although there are many causes of VTE 5,6 the present case was associated with pregnancy because the pelvic CT showed compression of the left common iliac vein by the enlarged uterus (Fig 1A). Although the incidence of VTE complication in the third trimester of pregnancy is less than $0.1 \%$, $^{5}$ the present patient had a high risk based on her serum proteinS concentration?

We used intermittent positive compression, standard heparin, 6,7 dalteparin, warfarin 6,7 and concomitant low-dose aspirin $^{10}$ to prevent the VTE progressing to APTE. Moreover, we inserted a t-IVC-f immediately before $\mathrm{C} / \mathrm{S}$ because of the high risk of APTE complication at the time of surgery? The postoperative clinical findings suggest APTE did not occur and lung perfusion scintigraphy on Day 8 after t-IVC-f insertion did not show any defects (Fig 2).

In the case of pregnancy-related VTE, the risk of APTE gradually decreases with time after $\mathrm{C} / \mathrm{S}$ ? In the present patient, venography of the IVC on Day 15 after t-IVC-f insertion did not show friable thrombus, and venography of the lower extremity showed no changes in VTE, which had also been noted on the venogram performed on Day 8 after insertion (Fig 2). Therefore, we decided to use the closed removal method for the t-IVC-f because of the risk of deterioration of the VTE and APTE in the future if the t-IVC-f remained inplace for a long period of time? The preferred removal method of a t-IVC-f that includes captured thrombus has not been established and there is a risk of inducing APTE at the time of removal through dispersion of thrombi, especially when the thrombus is fragile after fibrinolytic therapy or there is damage to the vascular wall when inserting and removing the filter. Therefore, in order to capture any dispersed thrombi, we inserted a second t-IVC-f immediately before closing the primary t-IVC-f, which contained the thrombus. Based on the morphology of the captured thrombus, there was a risk of inducing symptomatic APTE, and we consider that our method prevented the development of life-threatening APTE when removing the t-IVC-f and also prevented APTE during the peri-operative days of $\mathrm{C} / \mathrm{S}$.

The optimal duration of oral anticoagulant therapy in order to prevent the recurrence of APTE is also controversial ${ }^{11,12}$ and depends upon the patient-specific risk factors of VTE ${ }^{13}$ If the genesis of the idiopathic VTE in this case was pregnancy, then long-term anti-coagulant therapy is 
not essential. On the other hand, it is recommended that anticoagulant therapy be prolonged when the thrombosis is associated with persistent risk factors, ${ }^{14}$ and because the underlying reason for the present patient's edematous left leg has not been fully investigated, oral anticoagulant therapy has been continuous. According to Agnelli et al ${ }^{11}$ the risk of APTE does not increase for approximately 1 year after continuous oral anticoagulant therapy and so we hope to prevent the development of APTE.

In conclusion, this case indicates that a second t-IVC-f is useful for preventing APTE when removing a primary filter that contains captured friable thrombus. In addition, t-IVC-f is useful for preventing APTE in cases of pregnancyinduced VTE during the peri-operative days of C/S. Moreover, continuous oral anticoagulant therapy can prolong the benefit gained from the use of the filters.

\section{References}

1. Nakamura M, Fujioka H, Yamada N, Sakuma M, Okada O, Nakanishi N, et al. Clinical characteristics of acute pulmonary thromboembolism in Japan: Results of a multicenter registry in the Japanese Society of Pulmonary Embolism Research. Clin Cardiol 2001; 24: $132-138$.

2. Kumasaka N, Sakuma M, Shirato K. Incidence of pulmonary thromboembolism in Japan. Jpn Circ J 1999; 63: 439-441.

3. Decousus H, Leizorovicz A, Parent F, Page Y, Tardy B, Girard P, et al. A clinical trial of vena caval filters in the prevention of pulmonary embolism in patients with proximal deep-vein thrombosis. $N$ Engl $J$ Med 1998; 338: 409-415.

4. Heijboer H, Buller HR, Lensing WA, Turpie AGG, Colly LP, Cate JWT. A comparison of real-time compression ultrasonography with impedance plethysmography for the diagnosis of deep-vein thrombosis in symptomatic outpatients. N Engl J Med 1993; 329: $1365-$ 1369.

5. Gherman RB, Goodwin TM, Leung B, Byrne JD, Hethumumi R, Montoro M. Incidence, clinical characteristics, and timing of objectively diagnosed venous thromboembolism during pregnancy. Obstet Gynecol 1999; 94: 730-734.

6. Hirsh J, Hoak J. Management of deep vein thrombosis and pulmonary embolism. A statement for healthcare professionals. Circulation 1996; 93: 2212-2245.

7. Toglia MR, Weg JG. Venous thromboembolism during pregnancy. $N$ Engl J Med 1996; 335: 108-114.

8. Grehardt A, Scharf RE, Beckmann MW, Struve S, Bender HG, Pillny M, et al. Prothrombin and factor mutation in women with a history of thrombosis during pregnancy and the puerperium. $N$ Engl J Med 2000; 342: 374-380.

9. Sanson BJ, Lensing AW, Prins MH, Ginsberg JS, Barkagan ZS, Lavenne-Pardonge E, et al. Safety of low-molecular-weight heparin in pregnancy: A systematic review. Thromb Haemost 1999; 81: $668-672$.

10. Pulmonary Embolism Prevention (PEP) trial collaborative group. Prevention of pulmonary embolism and deep vein thrombosis with low dose aspirin: Pulmonary prevention (PEP) trial. Lancet 2000; 355: $1295-1302$.

11. Agnelli G, Prandoni P, Santamaria MG, Bagatella P, Iorio A, Bazzan $\mathrm{M}$, et al. Three months versus one year of oral anticoagulant therapy for idiopathic deep venous thrombosis. $N$ Engl J Med 2001; 345: $165-169$.

12. Tanimoto K, Mizushige K, Yukiiri K, Ueda T, Wada Y, Ohmori K, et al. Recurrence of idiopathic thromboembolism during anticoagluant therapy. Jpn Circ J 2001; 65: 755-756.

13. Hansson PO, Sorbo J, Eriksson H. Recurrence of venous thromboembolism after deep vein thrombosis: Incidence and risk factors. Arch Intern Med 2000; 160: 769-774.

14. Hyers TM, Agnelli G, Hull RD. Antithrombotic therapy for venous thromboembolic disease. Chest 2001; 119(Suppl): 176S-193S. 